\title{
Upcoming meetings
}

January 2013

SFAM Winter Meeting

January 9, 2013

London, UK

www.sfam.org.uk/en/events/index.cfm/Winter_meeting

February 2013

The Gut Microbiome:

The Effector/Regulatory Immune Network

February 10-15, 2013

Taos, NM USA

www.keystonesymposia.org/index.cfm? $\mathrm{e}=$ web. Meeting.

Program\&meetingid $=1231$

Biodefense and Emerging Diseases Research Meeting

February 25-27, 2013

Washington, DC USA

www.asmbiodefense.org

\section{March 2013}

Annual Conference of the Association for General and Applied Microbiology (VAAM)

March 10-13, 2013

Bremen, Germany

www.conventus.de/vaam-kongress/? $\mathrm{L}=1$

\section{April 2013}

1st International Probiotics Prebiotics

and Functional Food Congress

April 11-13, 201

Antalya, Turkey

www.ppd2013.org/eng

The 7th International Yakult Symposium/

The Intestinal Microbiota and Probiotics:

Exploiting Their Influence on Health

April 22-23, 2013

London, UK

www.yakultsymposium.com

23rd European Congress of Clinical Microbiology and Infectious Diseases

April 27-30, 2013

Berlin, Germany

www.congrex.ch/eccmid2013.html

\section{Positive Strand RNA Viruses}

April 28-May 3, 2013

Boston, MA USA

www.keystonesymposia.org/index.cfm? $=$ web.

Meeting.Program\&meetingid $=1230$

May 2013

Viruses and Cells

May 5-10, 2013

Lucca, Italy

www.grc.org/programs.aspx? year=2013\&program =viruses

\section{ASM 113th General Meeting}

May 18-21, 2013

Denver, CO USA

www.gm.asm.org

Microbiome and Host Health

May 12-14, 2013

Lisbon, Portugal

https://vicinity.picsrv.net/3186/

d32221faefee5e21be06cd416883cea0/2886

Probiotics and their Applications

May 31-June 1, 2013

Hanoi, Vietnam

http://probioticsconference.com

June 2013

International Scientific Conference on Probiotics and Prebiotics IPC2013

June 11-13, 2013

Kosice, Slovakia

www.probiotic-conference.net/Conference

ISAPP 2013:

11th Meeting of the International Scientific Association for Probiotics and Prebiotics, in collaboration

with the New York Academy of Sciences

and the Sackler Institute of Nutrition Sciences

June 12-14, 2013

New York, NY USA

www.nyas.org/Events/Detail.aspx?cid=c60ea8d5-44f0-

4aaa-a8ff-3e5f008186f6 


\section{June 2013 (continued)}

63rd Annual Conference of the Canadian

Society of Microbiologists

June 17-20, 2013

Ottawa, Canada

www.csm-scm.org/english/wn_meetings_det.asp?id=44

\section{CRISPR: Evolution, Mechanisms and Infection}

June 17-19, 2013

St. Andrews, UK

www.biochemistry.org/Conferences/AllConferences/tabid/379/

View/Conference/MeetingNo/SA148/Default.aspx

Swiss Society for Microbiology Annual Congress

June 26-27, 2013

Interlaken, Switzerland

www.swissmicrobiology.ch/Framesets/fr_annual_congress.htm

\section{July 2013}

\section{SFAM Summer Conference}

July 1-4, 2013

Cardiff, UK

www.sfam.org.uk/en/events/index.cfm/summer_conference

\section{Applied and Environmental Microbiology}

July 7-12, 2013

South Hadley, MA USA

www.grc.org/programs.aspx?year=2013\&program = applied

\section{ASM Adelaide 2013}

July 7-10, 2013

Adelaide, Australia

www.theasm.org.au/meetings/asm-adelaide-2013/

5th Congress of European Microbiologists

July 21-25, 2013

Leipzig, Germany

www2.kenes.com/fems2013/pages/home.aspx

\section{August 2013}

Society for Industrial Microbiology and Biotechnology Annual Meeting August 11-15, 2013, San Diego, CA USA www.simhq.org/annual

20th Biennial Evergreen International Phage Meetings August 11-16, 2013

Olympia, WA USA

http://blogs.evergreen.edu/phage/meetings/2013-2

\section{September 2013}

Probiotics, Prebiotics and New Foods

September 8-10, 2013

Rome, Italy

www.probiotics-prebiotics-newfood.org/home.php

ICAAC 2013

September 10-13, 2012

Denver, CO USA

www.icaac.org/index.php/scientific-program/meeting-resources/ icaac-online

\section{October 2013}

New Approaches and Concepts in Microbiology

October 14-16, 2013

Heidelberg, Germany

www.embo-embl-symposia.org/symposia/2013/EES13-05 